\title{
Epigenetics and Developmental Programming of Adult Onset Diseases
}

Lee O’Sullivan', Melissa H Little², Alexander N Combes² and Karen M Moritz ${ }^{1}$ ${ }^{1}$ School of Biomedical Sciences and ${ }^{2}$ Institute for Molecular Biosciences, The University of Queensland, St Lucia, 4072, Australia.

Address for correspondence:

Karen Moritz

School of Biomedical Sciences,

The University of Queensland,

St Lucia, 4067

Australia.

E-mail: k.moritz@uq.edu.au

Phone: 617-3365-4598

Fax: 61-7-3365-1299 


\begin{abstract}
:
Maternal perturbations or sub-optimal conditions during development are now recognized as contributing to the onset of many diseases manifesting in adulthood. This “developmental programming" of disease has been explored using animal models allowing insights into the potential mechanisms involved. Impaired renal development, resulting in a low nephron number, has been identified as a common outcome that is likely to contribute to the development of hypertension in the offspring as adults. Changes in other organs and systems including the heart and the hypothalamic-pituitaryadrenal axis (HPA) have also been found. Recently, evidence has emerged suggesting epigenetic changes may occur as a result of developmental programming and result in permanent changes in expression patterns of particular genes. Such epigenetic modifications may be responsible not only for an increased susceptibility to disease for an individual, but indirectly for the establishment of disease state in a subsequent generation. Further research in this field, particularly examination as to whether epigenetic changes to genes affecting kidney development occur, are essential to understanding underlying mechanisms of developmental programming of disease.
\end{abstract}

Key Words: kidney, glucocorticoids, prenatal programming, epigenetic modification 


\section{Introduction}

Hypertension is a major risk factor for the development of cardiovascular disease (CVD) and a common complication of other metabolic disorders such as obesity and type-2 diabetes. These chronic diseases are prevalent in society with more than $30 \%$ of adults in the U.S.A. having been diagnosed as suffering from hypertension [1, 2]. Subsequent to findings linking low birth weight to an increased risk of mortality from cardiovascular disease [3], it is widely accepted that the conditions in utero and early postnatal life play a role in a predisposition to adult disease by a process referred to as 'developmental programming' or the 'developmental origins of health and disease' [2, 4, 5]. However, the mechanisms by which an altered prenatal / perinatal milieu results in adult pathophysiology are incompletely understood.

Developmental programming of disease has been explored using animal models with studies demonstrating that many prenatal / early neonatal perturbations, including maternal dietary restrictions, placental insufficiency and excess glucocorticoid exposure, can result in low birth weight and the subsequent development of hypertension in the offspring [6]. Impaired development of the heart, kidney, brain and vasculature has been identified in the developing fetus following perturbation. In particular, the kidney seems to be exquisitely sensitive to insult during development with reduction in nephron endowment found in a variety of experimental models [6]. Similarly, such perturbations have also been shown to alter gene expression in a variety of affected organs. Whilst some of these gene changes are transient, other gene changes appear to persist and are thought to contribute to the disease phenotype. A suggested mechanism for such prolonged or permanent changes in gene expression occurring due to developmental 
programming is epigenetic modification of the genome [7, 8]. Such modifications enable alterations in the expression of genes to occur without any change in the DNA sequence. Epigenetic modifications may also be responsible for the transmission of a phenotype to a subsequent generation $[8,9]$. However, care must be taken to distinguish between an inherited epigenetic cause and the influence of an altered prenatal environment for any transgenerational phenotype. This review will encompass a discussion of the emerging experimental evidence that prenatal perturbations result in epigenetic changes with a focus on links to the development of a hypertensive phenotype.

\section{Prenatal programming of hypertension}

The concept that an individuals' risk of developing hypertension may be due, at least in part, to the environment they were exposed to in utero has been thoroughly explored in longitudinal human studies $[2,3,5]$ as well as in a wide variety of animal models $[2,4$, 6]. Human epidemiological studies have shown a correlation between birth weight and blood pressure in adulthood with a lower birth weight for gestational age being associated with increased blood pressure in later life. This association exists not just for babies with documented intrauterine growth restriction but across a range of normal birth weights [10]. Mechanisms underpinning the susceptibility to hypertension have been extensively investigated in animal models and include altered vascular reactivity [11, 12], reduced cardiomyocyte number in the heart [12], altered regulation of the hypothalamic-pituitaryadrenal (HPA) axis [13] and reductions in nephron endowment [6] (see Figure 1). Impaired renal development and a low nephron number is a strikingly common finding in a range of animal models highlighting the extreme vulnerability of the kidney to prenatal 
insult. Reductions in nephron endowment (and thus filtration surface area) are thought to limit the ability to excrete sodium resulting in an expansion of blood volume and ultimately hypertension as proposed by Brenner [14]. Reductions in nephron endowment may be compounded by changes in the renal renin-angiotensin system and sodium transporters within the kidney resulting in altered control of fluid and electrolyte balance (reviewed in 6). In some studies the nephron deficit alone is insufficient to cause hypertension, leading to the concept that a 'second hit', often a poor lifestyle (a high salt or high fat diet for example), is required to unmask disease susceptibility [6].

\section{What are epigenetic changes?}

DNA-encoded transcription factor binding sites play a critical role in regulating gene expression by enabling binding of proteins that activate or repress transcription at nearby loci. However, the presence of a genetically-encoded binding site and accompanying transcription factor are not the only factors that determine whether a gene is activated or repressed. Epigenetic modifications, such as DNA methylation or histone modifications, are involved in regulating gene activity by altering the structure or accessibility of a genomic region [15]. These modifications are considered to be 'epigenetic' because they affect transcription without changing the underlying nucleotide sequence. The most

common modifications of histone proteins include acetylation or methylation of individual amino acids, notably lysine (K)9 and K14 of the tail of the histone H3 protein. However histone proteins can also be modified via sumoylation, ubiquitinylation and phosphorylation [16, 17]. In general, increased histone aceytlation results in transcriptional activation, decreased acetylation results in transcriptional repression, 
whereas histone methylation can cause either repression or activation depending upon the gene locus involved. Such modifications are introduced by specific histone modifying enzymes, including histone acetyltransferases (HATs) in the case of acetylation. The other common epigenetic alteration is DNA methylation of cytosine to 5-methylsytosine, frequently within the cytosine-guanine rich $\mathrm{CpG}$ islands within the promoters of genes. Again, this is mediated by specific enzymes, the DNA methyltransferases, some of which are involved in the initial establishment of the DNA methylation pattern and others involved in maintenance of methylation. Methylation of the DNA sequence can result in local conformational changes that either increase or decrease the accessibility of that gene promoter to the proteins involved in regulating transcription from that locus. DNA methylation of promoter regions generally correlates with transcriptional repression [16, 17]. Thus, factors affecting methylation status have the potential to alter gene expression and subsequent development [8].

Epigenetic modifications are a necessary part of normal embryonic development. There is a major wave of DNA demethylation that occurs soon after fertilization followed by a wave of methylation in the blastocyst that is critical for resetting the imprinting of the genome. Epigenetic modifications to gene expression also play a key role in ensuring appropriate cellular differentiation such that only the required genes are expressed in a given cell type. Epigenetics is also required to compensate for gene dosage between males and females, a process called $\mathrm{X}$-inactivation, and in maintaining the allele specific expression of a set of 'imprinted' genes whose expression only occurs from a specific parental allele [18-20]. Once established, epigenetic modifications are stable throughout 
cell division, and thus are thought to be one way that cellular identity is maintained [21]. DNA methylation for example, is maintained throughout cell division by DNA methyltransferase 1 , which recognizes the methylation status of the template strand and replicates it on the newly synthesized strand $[22,23]$. Despite an understanding of the enzymes involved, little is known about what targets epigenetic modifications to specific sites in the genome. However, disruption to such processes is known to occur in disease states and has more recently been observed in association with perturbation of the environment during development. What remains to be determined is whether the epigenetic changes observed result from physiological perturbations, or do the epigenetic changes themselves induce these perturbations to occur.

\section{Are epigenetic marks inherited across generations?}

The epigenetic landscape of a genome is comprehensively re-set twice in a lifecycle; once in the germline, and again in the early embryo [21]. The first phase of reprogramming occurs when germ cells complete their migration through the early embryo to the developing gonads. The germ cells carry epigenetic information including paternal imprints that are established in the blastocyst [24]. After entering into the gonad, most genomic elements undergo a rapid loss of DNA methylation and repressive histone marks [25]. The one exception to this clearing event is retrovirus/retrotransposon-derived long terminal repeat regions (LTR), which retain a portion of their previous methylation, presumably to suppress activity of these elements $[23,26]$. The second phase of reprogramming occurs in the early embryo. Shortly after fertilization, the paternal and maternal haploid genomes are rapidly, but separately cleared of DNA methylation and 
undergo a raft of accompanying histone modifications [16]. However, again, some retrotransposons and imprinted sequences maintain their methylation through this process [27-29]. It is this dual genome-wide epigenetic reprogramming that hinders direct transmission of epigenetic information between generations.

\section{Evidence of epigenetic changes due to fetal programming}

Epigenetic changes have been found in both human and animal studies following perturbations in the prenatal environment. Whilst epigenetic changes and physiological changes have not generally been examined within a single study, it is possible to draw strong links between these outcomes from data reported in a number of animal models. In models of programmed hypertension, many epigenetic modifications occur within regulatory regions of genes influencing the HPA axis including the glucocorticoid receptor [30]. Epigenetic changes have also been found after induction of disease models in genes of the renin-angiotensin system (RAS) in the adrenal [31], as well as other genes in the kidney [32], pancreas [33, 34], liver [30, 35] and placenta [36]. A summary of these findings are shown in table 1. It should be noted that most of these findings are from relatively small cohorts and require subsequent validation in larger studies.

\section{Hypothalamic-pituitary-adrenal axis}

In humans, it has been demonstrated that maternal mood, in particular depression, can influence the methylation status of the $N R 3 C 1$ (glucocorticoid receptor) gene in DNA collected from umbilical cord blood. Infants of mothers who were classified as depressed during the third trimester of pregnancy showed increased methylation of the CpG3 
binding region of $\mathrm{NR} 3 \mathrm{Cl}$, which was associated with an increased response to stress, measured by salivary cortisol, at three months of age [37]. A very recent study has shown these prenatal effects may be long lasting; adolescents (10-19 years) of mothers who were subjected to violence during pregnancy had increased methylation of the glucocorticoid receptor [38].

In animal models, the HPA axis has been shown to be affected developmentally during both fetal and early neonatal environmental manipulation. Excess prenatal glucocorticoid exposure results not only in hypertension but an altered sensitivity of the HPA to stress (for review see [39]). In addition to the prenatal environment, the HPA axis has been shown to undergo induced epigenetic changes postpartum in rodents [40]. Maternal behaviour, characterised by licking, grooming and arched-back nursing (LG$A B N$ ) of their pups, was shown to influence the methylation status of the glucocorticoid receptor in the hippocampi of the offspring. Mothers classified as low-LG-ABN had offspring that had increased methylation of specific glucocorticoid receptor promoter DNA sequences compared to high-LG-ABN offspring. Cross fostering the pups within $12 \mathrm{~h}$ of birth to a mother in the opposite group resulted in the methylation status of the 5 , CpG dinucleotide in the offspring being identical to that of the biological offspring of the rearing mother. In other words, pups born to low-LG-ABN cross-fostered to high-LGABN mothers exhibited similar methylation status as pups born and reared by high-LGABN dams and vice versa. The difference in the response to a restraint stress was also significantly different between low-LG-ABN and high-LG-ABN offspring, with higher plasma corticosterone levels in the low-LG-ABN offspring. In addition, administration of the histone deacetylase inhibitor, trichostatin A (TSA), to the neonatal brain prevented 
the effects of the poor maternal behaviour. This experimental data elegantly illustrates the role that neonatal environment plays in shaping the epigenome and the impact a suboptimal environment can have on adult physiology.

Maternal alcohol consumption has also been identified as having a detrimental impact upon the development of the fetal HPA axis with effects on the stress response and hormonal systems persisting into adult life [41]. Recent experimental evidence linked prenatal alcohol exposure to epigenetic changes in the developing Agouti mouse [42] as in separate studies in the rat, to a nephron deficit and hypertension in offspring [43]. Hence, maternal alcohol consumption should be added to the list of maternal perturbations that have the possibility of causing epigenetic changes and hypertension in offspring.

\section{Kidney and adrenal}

Epigenetic changes have been seen in the kidney following uteroplacental insufficiency in the rat, including a decrease in $\mathrm{CpG}$ methylation of the $\mathrm{p} 53$ promoter resulting in increased expression of p53 mRNA levels. This was associated with increased apoptosis and a reduction in glomerular number [32]. In separate studies, this same maternal perturbation resulted in the development of hypertension [44]. Maternal undernutrition in the baboon has been shown to lead to decreased global DNA methylation in the kidney at mid gestation but increased methylation late in gestation [45]. The long term effects of such changes have yet to be examined.

Epigenetic changes in the RAS have been observed in the adrenals of offspring of rats fed on a low protein diet from conception. The type 1 angiotensin II receptor subtype 
b (AT1b) promoter was hypomethylated compared to control, resulting in increased AT1b mRNA expression at 12 weeks of age [46]. Interestingly, when a similar experiment was performed with the administration of metyrapone, an $11 \beta$-hydroxylase inhibitor that blocks the formation of corticosterone in the rat, for the first 14 days of pregnancy the methylation status of the AT1b promoter region was indistinguishable from control levels. Systolic blood pressure, which was elevated in the low protein diet offspring, was also returned to control levels with metyrapone administration [31]. This data highlights the susceptibility of the RAS to epigenetic change and suggests a glucocorticoid mediated role in RAS gene promoter methylation status. During development, the RAS is essential for normal kidney development and genetic deletion or mutation of any component of the system can lead to renal abnormalities [6]. Alterations in renal gene expression of components of the RAS have been consistently demonstrated in models of developmental programming including excess glucocorticoid exposure in the sheep [47] and rat [48], maternal protein restriction in the rat $[49,50]$ and mouse [51], as well as models of utero-placental insufficiency $[49,52]$. We have suggested that these changes in the RAS may contribute to the nephron deficit and the hypertensive phenotyope [6]. Whilst no studies have examined epigenetic changes in the RAS in kidneys following maternal perturbations, recently it has been shown that the ability of angiotensin II to induce branching morphogenesis in the kidney is epigenetically controlled via histone deacetylase activity [50].

In light of the evidence of epigenetic alterations of the RAS in the adrenal [31, 46], epigenetic modifications may account in some part for the changes in the RAS seen across a diverse range of experimental models. Given the critical role of the kidney in the 
programming of disease, this area warrants further investigation.

\section{Metabolic tissues}

Other organs and tissues which play a role in metabolic activity and regulation, such as the liver and pancreas, have also been shown to exhibit epigenetic modifications modifications in response to prenatal challenge. In a rat model of maternal protein restriction from conception until birth, the offspring had a $21 \%$ decrease in $\mathrm{CpG}$ methylation of the hepatic peroxisome proliferator-activated receptor $\alpha$ (PPAR $\alpha)$ promoter and a $23 \%$ decrease in the methylation of the hepatic glucocorticoid receptor promoter, with a corresponding increase in mRNA expression for both genes [35]. These genes influence carbohydrate and lipid metabolism which could have a profound impact upon glucose homeostasis and adiposity. This may in turn increase the risk of developing insulin resistance, type 2 diabetes or obesity, all key factors in the onset of hypertension. Strikingly, the methylation status of the promoter for $\operatorname{PPAR} \gamma_{1}$ (the dominant PPAR $\gamma$ isoform expressed in the liver) was unchanged, highlighting the specificity of DNA methylation for certain genes and their associated promoters rather than evoking a model in which there is a global change in methylation status. Lillycrop et al. (2007) [30] subsequently found a decrease in DNA methyltransferase type 1 (Dnmt1), a finding also observed in the kidney after a maternal low protein diet [32]. The reduction in the expression Dnmt1 might be a common mechanism allowing for the hypomethylation of specific promoters across many tissue types or organisms. A recent study in the baboon has shown maternal food restriction results in hypomethylation of the PCK1 promoter and increased PCK1 mRNA expression levels in the liver [53]. As PCK1 encodes for 
phosphoenolpyruvate carboxykinase 1 (PEPCK1), this epigenetic change resulted in increased PEPCK1 protein which may, in the long term, contribute to altered gluconeogenesis in offspring.

\section{Placenta}

Epigenetic changes in the placenta have also been found which may indirectly result in hypertensive offspring. These changes often relate to the imprinted insulin-like growth factor II (Igf2) gene. IGF2 is extremely important to both placental and fetal growth and lower expression of this gene as a result of epigenetic mechanisms may have lifelong consequences [54]. Of importance, the IGF2 gene itself, and adjacent genes such as $H 19$, fall within an imprinted control region such that these genes are normally only expressed off a specific parental allele. For example, IGF2 is only expressed from the paternally inherited (and methylated) IGF2 allele while $H 19$ is only expressed from the maternally inherited (and unmethylated) allele [55]. Overgrowth disorders such as BeckwithWiedemann syndrome [56], and cancers such as Wilms' tumour [57] are associated with the reactivation of the silenced parental allele of these genes. In humans, this specific imprinting is mediated via the imprinting control region 1, the region which regulates the expression of the $H 19$ and $I G F 2$ genes. The placentae of intrauterine growth restricted infants displayed an inappropriate hypomethylation of this region, which would result in suppression of the normally active paternal allele of IGF2 [36]. However, whole genome methylation was not affected nor were there any changes in the other imprinted loci on chromosome 11p15 [36]. Maternal ethanol exposure in the mouse resulted in a demethylation of the $H 19 / I G F 2$ domain compared to control causing both the embryo 
and the placentae to be growth retarded compared to control [58]. This suggests a particular susceptibility of the DNA in the IGF2 encoding region to hypomethylation in the developing placenta. This may represent a feedback loop to reduce IGF2-mediated growth in the presence of placental insufficiency [36].

\section{Do epigenetic changes explain intergenerational programming?}

At first glance it would appear that epigenetic modifications may play a principal role in programming of disease across generations [59]. However, as discussed above, most epigenetic marks are cleared and reset during early embryonic development. Therefore, persistence of an aberrant parental epigenetic state in the genome of the offspring requires that epigenetic state to escape reprogramming or be re-established after fertilisation in the next generation. In one example of intergenerational reprogramming, male offspring of females exposed to the synthetic glucocorticoid, dexamethasone, in utero, but who were subsequently left unmanipulated, exhibited a reduced birth weight and reduced glucose tolerance, as well having elevated levels of the hepatic gluconeogenic enzyme, PEPCK [59]. However, these changes did not persist into an F3 generation. Epigenetic changes to the endogenous glucocorticoid system have been seen in offspring whose prenatal environments were normal but whose neonatal environmental conditions were suboptimal [40]. Any change that resulted in offspring, particularly female offspring, having higher circulating glucocorticoid levels or an impaired glucocorticoid feedback mechanism persisting into adulthood clearly engenders a suboptimal intrauterine environment, with the potential to propagate fetal programming to the next generation. 
Although evidence of epigenetic modifications in the programming literature has been growing increasingly over the last few years, there is little evidence to support direct transmission of disease-causing epigenetic marks across generations. Furthermore, many of these studies have not been extensively followed up using larger cohorts to validate initial findings. A more likely model is the propagation of disease through suboptimal prenatal environment. Uteroplacental insufficiency has been reported to induce selective vascular dysfunction of the uterine vessels in female offspring at 18 months of age [11]. The animals were $10-15 \%$ growth restricted, but as adults were normotensive and had preserved function in their mesenteric, renal and femoral arteries. However, the females born small had increased stiffness, decreased smooth muscle relaxation and an increased deposition of thick collagen fibres in their uterine arteries. These alterations could clearly have an impact upon the ability to deliver nutrients and oxygen to any future developing fetus in the uterus, possibly leading to a second generation of growth restricted offspring. Whilst it is possible that the uteroplacental insufficiency offspring may also carry epigenetic modifications, cross-transfer experiments would be required to rule out the influence of the uterine environment on this phenotype in a subsequent generation.

\section{Conclusion}

Epigenetic modifications have been shown to impact upon the expression of key regulatory genes in organs and systems responsible for the maintenance of blood pressure, such as the kidney, RAS and HPA axis in animal models. There is also evidence that the human epigenome is susceptible to environmental influences. Epigenetic modifications contribute to lasting changes within an individual and can propagate 
disease states through creating a suboptimal uterine environment for the next generation. However, the molecular evidence for germline transmission of epigenetic modifications that have a direct bearing on fetal programming is lacking. The epigenetic modifications involved in prenatal programming of adult onset diseases, including hypertension and metabolic disease, are specific to the model used, the timing of the insult, the tissue, and gene of interest. Our understanding of the extent and role of these modifications will benefit from genome-wide studies of the changes involved. It has been demonstrated in the rat that disease-causing epigenetic changes brought about during early life can be circumvented either by a change to a more favourable environment (cross-fostering) or through pharmacological intervention [59].

Currently the vast majority of fetal programming studies have tended not to look at what role, if any, epigenetic alterations may have played in their phenotypic outcomes. This should become more of a focus given that multiple studies now present plausible evidence that epigenetic modifications influence and propagate developmental programming of disease. Care needs to be taken in designing robust experimental protocols including the validation of epigenetic modifications in large cohorts and following a range of prenatal insults. By doing so, our understanding of the mechanisms that lead to disease programming would be enhanced, and could reveal novel biomarkers present in individuals at risk, or new therapeutic options for the treatment of hypertension. 


\section{Bibliography}

1. Statistics NCfH, (COR) USDoHaHS (2011) Health, United States, 2010: With Special Feature on Death and Dying. p 547

2. Nuyt AM (2008) Mechanisms underlying developmental programming of elevated blood pressure and vascular dysfunction: evidence from human studies and experimental animal models. Clin Sci 114:1-17

3. Barker DJ, Osmond C (1986) Infant mortality, childhood nutrition, and ischaemic heart disease in England and Wales. Lancet 1:1077-1081

4. Gluckman PD, Hanson MA, Cooper C, Thornburg KL (2008) Effect of in utero and early-life conditions on adult health and disease. N Engl J Med 359:61-73

5. Huxley RR, Neil HAW (2004) Does maternal nutrition in pregnancy and birth weight influence levels of CHD risk factors in adult life? Br J Nutr 91:459-468.

6. Moritz KM, Wintour EM, Black MJ, Bertram JF, Caruana G (2008) Factors influencing mammalian kidney development: implications for health in adult life. Advances in anatomy, embryology, and cell biology 196:1-78

7. Pinney SE, Simmons RA (2010) Epigenetic mechanisms in the development of type 2 diabetes. Trends Endocrinol Metab 21:223-229

8. Drake AJ, Liu L (2010) Intergenerational transmission of programmed effects: public health consequences. Trends Endocrinol Metab 21:206-213

9. Harrison M, Langley-Evans SC (2009) Intergenerational programming of impaired nephrogenesis and hypertension in rats following maternal protein restriction during pregnancy. Br J Nutr 101:1020-1030

10. Barker DJP (2008) Human growth and cardiovascular disease. Nestlé Nut Workshop Series Pediatr Program 61:21-38

11. Mazzuca MQ, Wlodek ME, Dragomir NM, Parkington HC, Tare M (2010) Uteroplacental insufficiency programs regional vascular dysfunction and alters arterial stiffness in female offspring. J Physiol (Lond) 588:1997-2010

12. Bubb KJ, Cock ML, Black MJ, Dodic M, Boon WM, Parkington HC, Harding R, Tare M (2007) Intrauterine growth restriction delays cardiomyocyte maturation and alters coronary artery function in the fetal sheep. J Physiol (Lond) 578:871881

13. Shaltout HA, Chappell MC, Rose JC, Diz DI (2011) Exaggerated sympathetic mediated responses to behavioral or pharmacological challenges following antenatal betamethasone exposure. Am J Physiol Endocrinol Metab 300:E979985

14. Brenner BM, Garcia DL, Anderson S (1988) Glomeruli and blood pressure. Less of one, more the other? Am J Hypertens 1:335-347

15. Cedar H, Bergman Y (2009) Linking DNA methylation and histone modification: patterns and paradigms. Nat Rev Genet 10:295-304

16. Morgan HD, Santos F, Green K, Dean W, Reik W (2005) Epigenetic reprogramming in mammals. Hum Mol Genet 14 Spec No 1:R47-58.

17. Kouzarides T (2007) SnapShot: Histone-modifying enzymes. Cell 131:822

18. Cattanach BM, Beechey CV (1990) Autosomal and X-chromosome imprinting. Dev Suppl:63-72

19. Efstratiadis A (1994) Parental imprinting of autosomal mammalian genes. Curr Opin Genet Dev 4:265-280 
20. Radford EJ, Ferrón SR, Ferguson-Smith AC (2011) Genomic imprinting as an adaptative model of developmental plasticity. FEBS Lett 585:2059-2066

21. Reik W, Dean W, Walter J (2001) Epigenetic reprogramming in mammalian development. Science 293:1089-1093

22. Bestor TH, Ingram VM (1983) Two DNA methyltransferases from murine erythroleukemia cells: purification, sequence specificity, and mode of interaction with DNA. Proc Natl Acad Sci USA 80:5559-5563

23. Yoder JA, Soman NS, Verdine GL, Bestor TH (1997) DNA (cytosine-5)methyltransferases in mouse cells and tissues. Studies with a mechanism-based probe. J Mol Biol 270:385-395

24. Sasaki H, Matsui Y (2008) Epigenetic events in mammalian germ-cell development: reprogramming and beyond. Nat Rev Genet 9:129-140

25. Hajkova P, Ancelin K, Waldmann T, Lacoste N, Lange UC, Cesari F, Lee C, almouzni G, Schneider R, Surani MA (2008) Chromatin dynamics during epigenetic reprogramming in the mouse germ line. Nature 452:877-881

26. Popp C, Dean W, Feng S, Cokus SJ, Andrews S, Pellegrini M, Jacobsen SE, Reik W (2010) Genome-wide erasure of DNA methylation in mouse primordial germ cells is affected by AID deficiency. Nature 463:1101-1105

27. Lane N, Dean W, Erhardt S, Hajkova P, Surani A, Walter J, Reik W (2003) Resistance of IAPs to methylation reprogramming may provide a mechanism for epigenetic inheritance in the mouse. Genesis 35:88-93

28. Edwards CA, Ferguson-Smith AC (2007) Mechanisms regulating imprinted genes in clusters. Curr Opin Cell Biol 19:281-289

29. Blewitt ME, Gendrel A-V, Pang Z, Sparrow DB, Whitelaw N, Craig JM, Apedaile A, Hilton DJ, Dunwoodie SL, Brockdorff N, Kay GF, Whitelaw E (2008) SmcHD1, containing a structural-maintenance-of-chromosomes hinge domain, has a critical role in X inactivation. Nat Genet 40:663-669

30. Lillycrop KA, Slater-Jefferies JL, Hanson MA, Godfrey KM, Jackson AA, Burdge GC (2007) Induction of altered epigenetic regulation of the hepatic glucocorticoid receptor in the offspring of rats fed a protein-restricted diet during pregnancy suggests that reduced DNA methyltransferase-1 expression is involved in impaired DNA methylation and changes in histone modifications. Br J Nutr 97:1064-1073

31. Bogdarina IG, Haase A, Langley-Evans SC, Clark AJL (2010) Glucocorticoid effects on the programming of AT1b angiotensin receptor gene methylation and expression in the rat. PLoS ONE 5:e9237

32. Pham TD, MacLennan NK, Chiu CT, Laksana GS, Hus JL, Lane RH (2003) Uteroplacental insufficiency increases apoptosis and alters p53 gene methylation in the full-term IUGR rat kidney. Am J Physiol Regul Integr Comp Physiol 285:R962-970

33. Park JH, Stoffers DA, Nicholls RD, Simmons RA (2008) Development of type 2 diabetes following intrauterine growth retardation in rats is associated with progressive epigenetic silencing of Pdx1. Journal Clin Invest 118:2316-2324

34. Sandovici I, Smith NH, Nitert MD, Ackers-Johnson M, Uribe-Lewis S, Ito Y, Jones RH, Marquez VE, Cairns W, Tadayyon M, O’Neill LP, Murrell A, Ling C, Constância M, Ozanne SE (2011) Maternal diet and aging alter the epigenetic 
control of a promoter-enhancer interaction at the $\mathrm{Hnf} 4 \mathrm{a}$ gene in rat pancreatic islets. Proc Natl Acad Sci U S A 108:5449-5454

35. Lillycrop KA, Phillips ES, Jackson AA, Hanson MA, Burdge GC (2005) Dietary protein restriction of pregnant rats induces and folic acid supplementation prevents epigenetic modification of hepatic gene expression in the offspring. $\mathrm{J}$ Nutr 135:1382-1386

36. Bourque DK, Avila L, Peñaherrera M, von Dadelszen P, Robinson WP (2010) Decreased placental methylation at the H19/IGF2 imprinting control region is associated with normotensive intrauterine growth restriction but not preeclampsia. Placenta 31:197-202

37. Oberlander TF, Weinberg J, Papsdorf M, Grunau R, Misri S, Devlin AM (2008) Prenatal exposure to maternal depression, neonatal methylation of human glucocorticoid receptor gene (NR3C1) and infant cortisol stress responses. Epigenetics 3:97-106

38. Radtke K, Ruf M, Gunter H, Dohrmann K, Schauer M, Meyer A, Elbert T (2011)

Transgenerational impact of intimate partner violence on methylation in the promoter of the glucocorticoid receptor. Transl Psychiatry

DOI:10.1038/tp.2011.21

39. Cottrell EC, Seckl JR (2009) Prenatal stress, glucocorticoids and the programming of adult disease. Front Behav Neurosci 3:19

40. Weaver ICG, Cervoni N, Champagne FA, D'Alessio AC, Sharma S, Seckl JR, Dymov S, Szyf M, Meaney MJ (2004) Epigenetic programming by maternal behavior. Nat Neurosci 7:847-854

41. Weinberg J, Sliwowska JH, Lan N, Hellemans KG (2008) Prenatal alcohol exposure: foetal programming, the hypothalamic-pituitary-adrenal axis and sex differences in outcome. J Neuroendocrinol 20:470-488

42. Kaminen-Ahola N, Ahola A, Maga M, Mallitt KA, Fahey P, Cox TC, Whitelaw E, Chong S (2010) Maternal ethanol consumption alters the epigenotype and the phenotype of offspring in a mouse model. PLoS Genet 6:e1000811

43. Gray SP, Denton KM, Cullen-McEwen L, Bertram JF, Moritz KM (2010) Prenatal exposure to alcohol reduces nephron number and raises blood pressure in progeny. J Am Soc Nephrol 21:1891-1902

44. Wlodek ME, Westcott K, Siebel AL, Owens JA, Moritz KM (2008) Growth restriction before or after birth reduces nephron number and increases blood pressure in male rats. Kidney Int 74:187-195

45. Unterberger A, Szyf M, Nathanielsz PW, Cox LA (2009) Organ and gestational age effects of maternal nutrient restriction on global methylation in fetal baboons. $\mathrm{J}$ Med Primatol 38:219-227

46. Bogdarina IG, Welham S, King PJ, Burns SP, Clark AJ (2007) Epigenetic modification of the renin-angiotensin system in the fetal programming of hypertension. Circ Res 100:520-526

47. Moritz KM, Johnson K, Douglas-Denton R, Wintour EM, Dodic M (2002) Maternal glucocorticoid treatment programs alterations in the renin-angiotensin system of the ovine fetal kidney. Endocrinology 143:4455-4463

48. Singh RR, Cullen-McEwen LA, Kett MM, Boon WM, Dowling J, Bertram JF, Moritz KM (2007) Prenatal corticosterone exposure results in altered AT1/AT2, 
nephron deficit and hypertension in the rat offspring. J Physiol (Lond) 579:503513

49. Moritz KM, Mazzuca MQ, Siebel AL, Mibus A, Arena D, Tare M, Owens JA, Wlodek ME (2009) Uteroplacental insufficiency causes a nephron deficit, modest renal insufficiency but no hypertension with ageing in female rats. J Physiol (Lond) 587:2635-2646

50. Song R, Van Buren T, Yosypiv IV (2010) Histone deacetylases are critical regulators of the renin-angiotensin system during ureteric bud branching morphogenesis. Pediatr Res 67:573-578

51. Hoppe CC, Evans RG, Bertram JF, Moritz KM (2007) Effects of dietary protein restriction on nephr Moritz KM, Mazzuca MQ, Siebel AL on number in the mouse. Am J Physiol Regul Integr Comp Physiol 292:R1768-1774.

52. Grigore D, Ojeda NB, Robertson EB, Dawson AS, Huffman CA, Bourassa EA, Speth RC, Brosnihan KB, Alexander BT (2007) Placental insufficiency results in temporal alterations in the renin angiotensin system in male hypertensive growth restricted offspring. Am J Physiol Regul Integr Comp Physiol 293:R804-811

53. Nijland MJ, Mitsuya K, Li C, Ford S, McDonald TJ, Nathanielsz PW, Cox LA (2010) Epigenetic modification of fetal baboon hepatic phosphoenolpyruvate carboxykinase following exposure to moderately reduced nutrient availability. J Physiol (Lond) 588:1349-1359

54. Constância M, Hemberger M, Hughes J, Dean W, Ferguson-Smith A, Fundele R, Steward F, Kelsey G, Fowden A, Sibley C, Reik W(2002) Placental-specific IGFII is a major modulator of placental and fetal growth. Nature 417:945-948

55. Murrell A, Heeson S, Reik W (2004) Interaction between differentially methylated regions partitions the imprinted genes Igf 2 and H19 into parent-specific chromatin loops. Nat Genet 36:889-893

56. Schneid H, Seurin D, Vazquez MP, Gourmelen M, Cabrol S, Le Bouc Y (1993) Parental allele specific methylation of the human insulin-like growth factor II gene and Beckwith-Wiedemann syndrome. J Med Genet 30:353-362

57. Steenman MJ, Rainier S, Dobry CJ, Grundy P, Horon IL, Feinberg AP (1994) Loss of imprinting of IGF2 is linked to reduced expression and abnormal methylation of H19 in Wilms' tumour. Nat Genet 7:433-439

58. Haycock PC, Ramsay M (2009) Exposure of mouse embryos to ethanol during preimplantation development: effect on DNA methylation in the h19 imprinting control region. Biol Reprod 81:618-627

59. Drake AJ, Walker BR, Seckl JR (2005) Intergenerational consequences of fetal programming by in utero exposure to glucocorticoids in rats. Am J Physiol Regul Integr Comp Physiol 288:R34-38 
Table 1: Some reported epigenetic changes following prenatal insults in humans and models of developmentally programmed disease

Epigenetic change
Changes in the glucocorticoid receptor/promoter

Increased methylation

Increased methylation

Increased methylation

Decreased methylation of GR1(10)promoter

Changes in kidney/adrenal

P53 promoter

Decreased methylation of the

AT1b receptor

Changes in genes regulation metabolism

Decreased methylation of PPAR $\alpha$

Decreased methylation of PCK1 promoter

Changes in the placenta

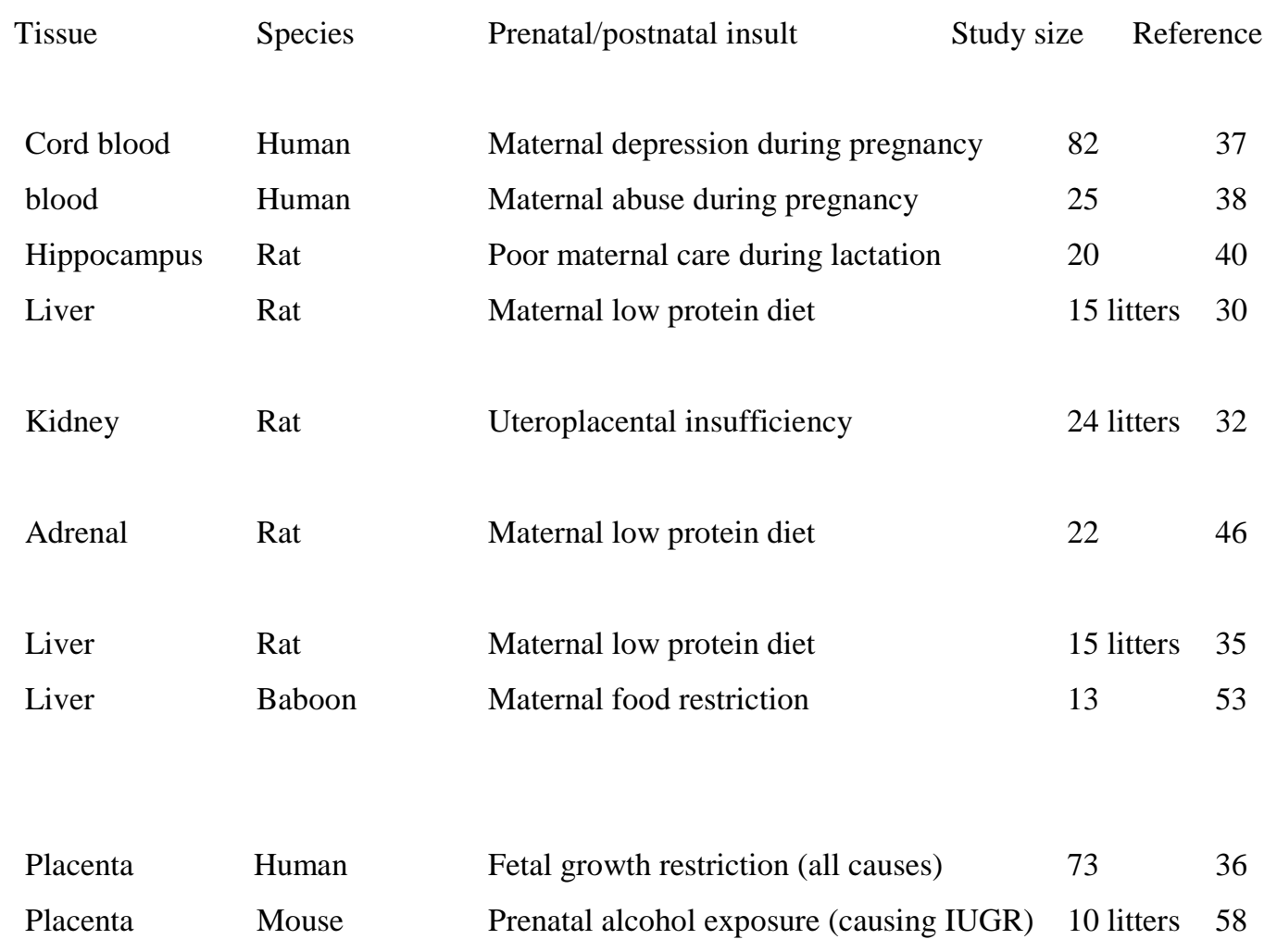

Hypomethylation of the imprinting

Control region - 1 (ICR1)

Demethylation of H19/IGF2 domain

Placenta Mouse

Prenatal alcohol exposure (causing IUGR) 10 litters 58 


\section{Figure Legend:}

Figure 1: Representative overview of how in utero environmental stressors on the fetus have been implicated in the development of hypertension, chronic vascular disease and chronic kidney disease during adulthood. Both physiological changes in organ function and development together with molecular changes affecting the epigenetic status of key genetic pathways have been recorded, however the etiology of these changes and how they are interrelated remains unclear. 


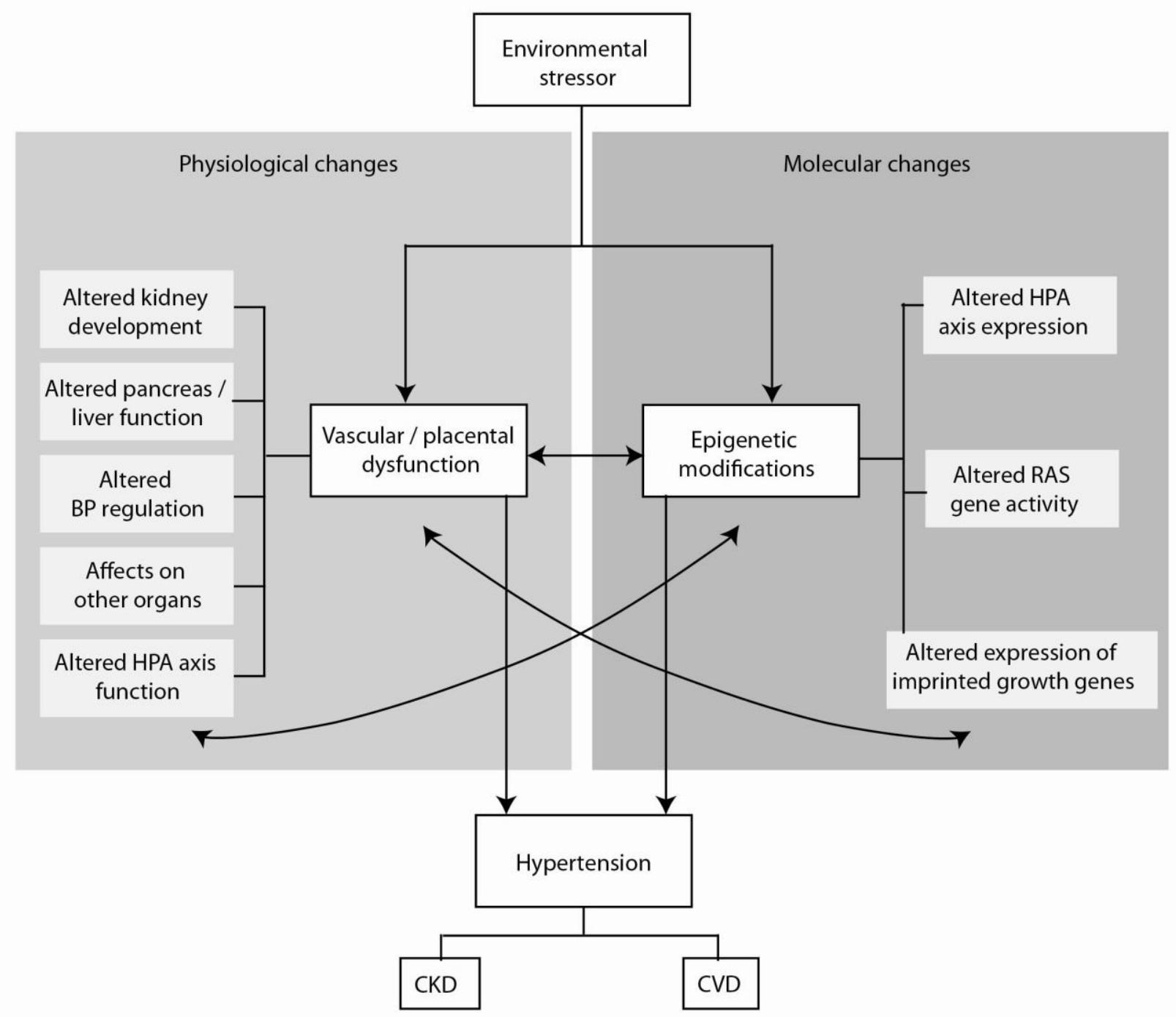

Figure 1: Representative overview of how in utero environmental stressors on the fetus have been implicated in the development of hypertension, chronic vascular disease and chronic kidney disease during adulthood. Both physiological changes in organ function and development together with molecular changes affecting the epigenetic status of key genetic pathways have been recorded, however the etiology of these changes and how they are interrelated remains unclear. 


\section{University Library}

\section{- M M N E R VA A gateway to Melbourne's research publications}

Minerva Access is the Institutional Repository of The University of Melbourne

Author/s:

O'Sullivan, L;Little, MH;Combes, AN;Moritz, KM

Title:

Epigenetics and developmental programming of adult onset diseases

Date:

2012-12-01

Citation:

O'Sullivan, L., Little, M. H., Combes, A. N. \& Moritz, K. M. (2012). Epigenetics and

developmental programming of adult onset diseases. PEDIATRIC NEPHROLOGY, 27 (12), pp.2175-2182. https://doi.org/10.1007/s00467-012-2108-x.

Persistent Link:

http://hdl.handle.net/11343/283085 